\title{
$B$-meson production in the general-mass variable-flavour-number scheme and LHC data
}

\author{
M. Benzke ${ }^{1}$, B. A. Kniehl ${ }^{2}$, G. Kramer ${ }^{1}$, I. Schienbein ${ }^{3}$, H. Spiesberger ${ }^{4,5, a}$ \\ ${ }^{1}$ II. Institut für Theoretische Physik, Universität Hamburg, Luruper Chaussee 149, 22761 Hamburg, Germany \\ ${ }^{2}$ University of California at San Diego, 9500 Gilman Drive, La Jolla, CA 92093, USA \\ ${ }^{3}$ Laboratoire de Physique Subatomique et de Cosmologie, Université Joseph Fourier Grenoble 1, CNRS/IN2P3, Institut National Polytechnique \\ de Grenoble, 53 Avenue des Martyrs, 38026 Grenoble, France \\ ${ }^{4}$ PRISMA+ Cluster of Excellence, Institut für Physik, Johannes-Gutenberg-Universität, Staudinger Weg 7, 55099 Mainz, Germany \\ ${ }^{5}$ Centre for Theoretical and Mathematical Physics and Department of Physics, University of Cape Town, Rondebosch 7700, South Africa
}

Received: 8 August 2019 / Accepted: 19 September 2019 / Published online: 3 October 2019

(C) The Author(s) 2019

\begin{abstract}
We study inclusive $B$-meson production in $p p$ collisions at the LHC and compare experimental data with predictions of the general-mass variable-flavour-number scheme at next-to-leading order of perturbative QCD. We find almost perfect agreement provided that the factorization scale parameters and the parton distribution functions are chosen appropriately.
\end{abstract}

\section{Introduction}

The study of the inclusive production of hadrons containing $b$ quarks, as for example $B^{ \pm}, B^{0}, \bar{B}^{0}, B_{s}^{0}, \bar{B}_{s}^{0}$ mesons and $\Lambda_{b}^{0}$ baryons, is particularly important to test quantum chromodynamics (QCD). The predictions in the framework of perturbative QCD are based on the factorization approach. In this approach, the production cross section is calculated as a convolution of three basic ingredients: the parton distribution functions (PDFs) describing the parton content of the hadronic initial state, the partonic hard-scattering cross section computed as a perturbative series in powers of the strong-coupling constant, and the fragmentation functions (FFs), which describe the momentum distribution for specified $b$ hadrons in a parton. Since the $b$-quark mass is large and cannot be neglected in the small transverse momentum region, the cross section for $b$-hadron production depends on several large scales, which makes predictions of the cross sections very demanding.

B. A. Kniehl: On leave of absence from II. Institut für Theoretische Physik, Universität Hamburg, Luruper Chaussee 149, 22761 Hamburg, Germany.

a e-mail: spiesber@uni-mainz.de
In the past, measurements of inclusive $b$-hadron production and the corresponding perturbative QCD calculations have been performed for all of the $B$ mesons mentioned above, and for $\Lambda_{b}^{0}$ baryons. Some time ago, data for $p \bar{p}$ collisions at center-of-mass energy $\sqrt{S}=1.96 \mathrm{TeV}$ have been obtained at the FNAL Tevatron Collider by the CDF Collaboration [1,2] and later for $p p$ collisions at $\sqrt{S}=5,7,8$ and $13 \mathrm{TeV}$ at the CERN Large Hadron Collider (LHC) by the ATLAS, CMS and LHCb Collaborations [3-10]. First measurements of the inclusive production cross sections of $\Lambda_{b}^{0}$ baryons have been presented by the CMS Collaboration [11] for $\sqrt{S}=7 \mathrm{TeV}$ and by the LHCb Collaboration for $\sqrt{S}=7$ and $8 \mathrm{TeV}$ [12].

Almost all these data have been compared with next-toleading-order (NLO) QCD predictions based on the so-called fixed-order next-to-leading-logarithmic (FONLL) approach [13]. Data of the CMS, LHCb and ATLAS Collaborations have also been compared with predictions obtained in the general-mass variable-flavour-number scheme (GM-VFNS) $[14,15]$. The GM-VFNS as originally formulated in Refs. $[16,17]$ (see also Ref. [18] for a more recent implementation of the GM-VFNS including its application to charm meson production) is similar to the FONLL scheme, but incorporates different assumptions concerning fragmentation functions, and the transition to the fixed-flavour-number scheme (FFNS) in the low transverse momentum region is treated in a different way. All comparisons between experimental data and theoretical predictions, both in the FONLL and GM-VFNS approaches, have shown reasonable agreement within experimental errors and the theoretical uncertainty, which is usually estimated by a variation of the factorization and renormalization scale parameters and the bottom quark mass. 
For large transverse momenta $p_{T}$, say $p_{T} \gtrsim 8 \mathrm{GeV}$, the two approaches yield approximately the same differential cross sections $d \sigma / d p_{T}$ as a function of $p_{T}$. Most of the data from the CMS and ATLAS Collaborations lie in this $p_{T}$ region. Comparisons with GM-VFNS predictions can be found in Refs. $[14,15]$. Data in the low $p_{T}$ region, i.e. for $0<p_{T}<8 \mathrm{GeV}$, have been obtained by the LHCb Collaboration $[19,20]$ and also by the CDF Collaboration $[1,2]$, although at different $\sqrt{S}$ values. Tevatron data exist at $\sqrt{S}=1.96 \mathrm{TeV}$, while data from the LHCb Collaboration are obtained at $\sqrt{S}=7 \mathrm{TeV}[19,20]$ and at $\sqrt{S}=13 \mathrm{TeV}$ [20]. Also the rapidity $(y)$ range in these experiments is different: $|y| \leq 1$ for the CDF measurements [1,2] and $2.0<y<4.5$ for the two LHCb measurements $[19,20]$. The behaviour of $d \sigma / d p_{T}$ as a function of $p_{T}$ in the low and the high $p_{T}$ ranges is quite different. At large $p_{T}, d \sigma / d p_{T}$ is monotonically decreasing in accordance with the expected behaviour originating from the interplay of the momentum dependence of the hard-scattering cross section and the scale dependence of the PDFs and FFs. In the low $p_{T}$ region, $0<p_{T}<8 \mathrm{GeV}, d \sigma / d p_{T}$ behaves quite differently, both for the $p \bar{p}$ data from CDF $[1,2]$ and the $p p$ data from LHCb $[19,20]$. Towards low $p_{T}$, the heavy-quark production threshold takes over and $d \sigma / d p_{T}$ reaches a maximum at $p_{T} \simeq 2.5 \mathrm{GeV}$ and then decreases towards $p_{T}=0$.

This behaviour is very well predicted theoretically within the FFNS as shown in previous work [15]. There we have also shown how the FFNS results at small $p_{T}$ can be incorporated in the GM-VFNS by choosing appropriate factorization scales. We use the notation $\mu_{i}$ for the initial-state factorization scale entering the PDFs and $\mu_{f}$ for the finalstate factorization scale entering the FFs. In Ref. [15] these two scales were fixed at the same value, $\mu_{i}=\mu_{f}=\mu_{F}:=$ $0.5 \sqrt{p_{T}^{2}+m_{b}^{2}}$ with $m_{b}=4.5 \mathrm{GeV}$. This leads to a suppression of the contribution of the $b$-quark PDF in the proton for $p_{T} \simeq 8 \mathrm{GeV}$ due to the threshold $\mu_{\mathrm{thr}}=m_{b}$ as implemented in both the PDFs and FFs chosen in Ref. [15], and we could describe the CDF data $[1,2]$ very well in the $p_{T}$ range $0<p_{T}<25 \mathrm{GeV}$ and the LHCb data fairly well in the $p_{T}$ range $0<p_{T}<12 \mathrm{GeV}$ showing a maximum of $d \sigma / d p_{T}$ near $p_{T} \simeq 2.5 \mathrm{GeV}$.

In Ref. [15], we used factorization scales in such a way that the transition to the FFNS occurs at rather large $p_{T}$ values. This choice was satisfactory: the predictions of the GM-VFNS were found to be consistent with the data at $\sqrt{S}=7 \mathrm{TeV}$ inside the theoretical error estimated by varying the renormalization scale by a factor of two. In the present work, we attempt to improve the agreement between $\mathrm{LHCb}$ data and predictions, in particular in the low $p_{T}$ region, by using a more general ansatz for the factorization scale, which produces the transition to the FFNS at much smaller $p_{T}$ values. This new ansatz will be described in the next section. It was used already in the calculation of charm-meson production in the same kinematical region as for $B^{ \pm}$-meson production in the present work, and it was successfully compared with the relevant LHCb [21], ALICE and CDF data [22].

In the present work, we shall compare with the more recent LHCb measurements at $\sqrt{S}=7$ and $13 \mathrm{TeV}$ [20]. These measurements extend all the way up to $p_{T}=40 \mathrm{GeV}$. The cross section data in five rapidity bins in the forward region $2.0<y<4.5$ are much more accurate than the previous data at $\sqrt{S}=7 \mathrm{TeV}$ [19]. We hope that a comparison of predictions for cross sections and cross section ratios with these 7 and $13 \mathrm{TeV}$ data will allow us to obtain additional information on the proton PDFs at small $x$ values.

The outline of our work is as follows. In Sect. 2, we describe the setup of our calculation aiming at a comparison with the recent $\mathrm{LHCb} \mathrm{B}^{+}$-production data and discuss in particular the possible choices of PDFs. In Sect. 3, we present numerical results and a comparison with the $\mathrm{LHCb}$ data [20]. We start with the cross sections $d^{2} \sigma / d p_{T} d y$ for $\sqrt{S}=7$ and $13 \mathrm{GeV}$ for the five rapidity bins in the range $2.0<y<4.5$ as a function of $p_{T}$ in the range between 0 and $40 \mathrm{GeV}$. Then we study the single-differential cross section $d \sigma / d p_{T}$ as a function of $p_{T}$ in the same $p_{T}$ range, but summed over the five $y$ bins, i.e. for $2.0<y<4.5$, and calculate from them the ratio of the cross sections for $\sqrt{S}=13$ and $7 \mathrm{TeV}$. Finally, we also present the rapidity dependence of the cross section, $d \sigma / d y$ integrated over $p_{T}$ in the range $0<p_{T}<40 \mathrm{GeV}$ and consider the $13 \mathrm{TeV}$ to $7 \mathrm{GeV}$ ratio. In Sect. 4, we present a summary and some concluding remarks.

\section{Setup and input}

The theoretical description of the GM-VFNS approach as well as various technical details of its implementation have been presented in our previous papers [16,17]. Here we describe only the input for the numerical evaluation discussed below, in particular our choice of the proton PDF.

As a default, we use the PDF set CT14 [23] at NLO. Other choices are (i) HERAPDF2.0 [24], (ii) MMHT [25], (iii) NNPDF3.1 [27]. All these PDF sets are NLO parametrizations; the last two of them are obtained from global fits to essentially the same experimental data as CT14, while HERAPDF2.0 is based on cross section data from deep inelastic scattering at HERA only. All PDF parametrizations have been taken from their implementation in the LHAPDF library [28].

To describe the transition of $b$ quarks to $B^{+}$mesons, we need nonperturbative FFs. We use the $B$-meson FFs constructed in Ref. [29]. They are evolved at NLO, and all components for the transition from gluons and light quarks to a $B$ meson are generated through the Dokshitzer-Gribov- 
Lipatov-Altarelli-Parisi evolution. They were obtained by fitting experimental data for inclusive $b$-hadron production in $e^{+} e^{-}$annihilation taken by the ALEPH [30] and OPAL [31] Collaborations at CERN LEP1 and by the SLD Collaboration [32,33] at SLAC SLC. These data were all taken at the $Z$-boson resonance. Therefore the strong coupling $\alpha_{s}^{\left(n_{f}\right)}\left(\mu_{R}\right)$ was calculated with $n_{f}=5$ active quark flavors and the renormalization and factorization scales were fixed at $\mu_{R}=\mu_{F}=m_{Z}$. The starting scale was chosen to be $\mu_{0}=m_{b}=4.5 \mathrm{GeV}$. Below $\mu_{F}=\mu_{0}$, the lightquark (including charm) FFs and the gluon FF were assumed to vanish. A simple power ansatz for the $b$-quark FF at $\mu_{0}$ gave the best fit to the experimental data. Recently also FFs at NNLO have become available [34]. They were obtained from fits which also include $b$-hadron production data from the DELPHI Collaboration [35].

The essential ingredient in the numerical calculations presented below is the choice of the factorization scales $\mu_{F}=\mu_{i}=\mu_{f}$. They are fixed by

$\mu_{F}=0.49 \sqrt{p_{T}^{2}+4 m_{b}^{2}}$

with the heavy-quark mass $m_{b}=4.5 \mathrm{GeV}$. This value of $m_{b}$ equals the threshold of the $b \rightarrow B^{+} \mathrm{FF}$. The scale choice in Eq. (1) is similar to the one used in Refs. [21,22], where a successful interpretation of LHCb, ALICE and CDF data was achieved. The factor 4 in front of $m_{b}^{2}$ can also be justified by the fact that the dominant LO contribution in the FFNS originates from the channel $g g \rightarrow b \bar{b}$ which has the threshold $2 m_{b}$. Both for PDFs and FFs we keep $\mu_{i}$ and $\mu_{f}$ constant below the threshold value $\mu_{0}=m_{b}$, i.e. the PDFs and FFs are not evolved when $p_{T}$ is further decreased and $\mu_{F}$, as a function of $p_{T}$, becomes smaller than $m_{b}$. With the definition in Eq. (1) this always happens at $p_{T}=1.83 \mathrm{GeV}$. The renormalization scale $\mu_{R}$, however, is allowed to vary with $p_{T}$ also below the threshold.

In general, one would argue that the threshold values in the PDFs and the FFs should be the same. On the other hand, both PDFs and FFs are non-perturbative objects which are determined by fits to experimental data. Therefore, the threshold values $m_{b}^{\text {(thr) }}$ used in the PDFs are also fixed by data, and it may turn out that different values are needed in PDFs and FFs. In addition, the parameter $m_{b}$ in Eq. (1) is not necessarily the same as either of the two threshold values in the FFs and PDFs. In our numerical evaluations, we shall always assume $m_{b}=4.5 \mathrm{GeV}$ in the definition of the renormalization and factorization scales, Eq. (1), i.e. equal to the threshold value used in the FFs. With our common choice for $m_{b}$ in $\mu_{i}$, but differing threshold values $m_{b}^{\text {(thr) }}$ in the PDFs, the turn-on of the $b$ component takes place at different transverse momenta. Initial-state $b$ quarks contribute only if $\mu_{i}\left(p_{T}\right)>m_{b}^{\text {(thr) }}$, i.e. if the transverse momentum is large enough, while at small $p_{T}$ the $b$ component is suppressed. For CT14 and
MMHT with $m_{b}^{\text {(thr) }}=4.75 \mathrm{GeV}$, we have $\mu_{i}>m_{b}^{\text {(thr) }}$ for $p_{T} \geq 3.60 \mathrm{GeV}$, for HERAPDF2.0 with $m_{b}^{\text {(thr) }}=4.5 \mathrm{GeV}$ the transition already occurs at $p_{T} \geq 1.83 \mathrm{GeV}$ and for the recent set NNPDF3.1, where $m_{b}^{\text {(thr) }}=4.92 \mathrm{GeV}$ is used, we find the threshold at $p_{T} \geq 4.45 \mathrm{GeV}$. For the CT14, MMHT and NNPDF3.1 sets, the $b$ component of the proton PDF vanishes for small enough $p_{T}$ values with the scale choice in Eq. (1).

The influence of the different PDF sets on $d \sigma / d p_{T}$ integrated over the rapidity range $2.0<y<4.5$ is shown in Fig. 1. In the left panel of this figure, the predictions for $d \sigma / d p_{T}$ obtained using the three PDF sets, CT14, MMHT, HERAPDF2.0 and NNPDF3.1, are compared for $0 \leq p_{T} \leq$ $40 \mathrm{GeV}$ as a function of $p_{T}$. The full black curve is for CT14, which agrees very well with the LHCb data [20]. The comparison with data will be discussed in more detail in the next section. The results for MMHT and NNPDF3.1 are very close to the CT14 curve and also agree with the LHCb data. All three predictions of $d \sigma / d p_{T}$ agree with each other for $p_{T} \geq 10 \mathrm{GeV}$, as expected. Although all three predictions for $d \sigma / d p_{T}$ exhibit maxima approximately at the same $p_{T}$ values due to the suppression of the incoming $b$-quark contribution, the $p_{T}$ values of the maxima differ appreciably. For HERAPDF2.0 they are approximately three times larger than for CT14. This difference originates from the much smaller $p_{T}$ value at which the initial $b$-quark contribution of the proton decouples in the case of HERAPDF2.0.

The contribution due to $b$ quarks in the initial state can be inferred from the results shown in the right part of Fig. 1. There we have plotted $d \sigma / d p_{T}$ for CT14 including all contributions (black full-line histogram, the same as in the left part Fig. 1) and with the contribution from incoming $b$ quarks subtracted (dashed red histogram). In the region $p_{T} \lesssim 4 \mathrm{GeV}$, the two histograms coincide, whereas for $p_{T} \gtrsim 4 \mathrm{GeV}$ one can see that the contribution from incoming $b$ quarks becomes more and more important. Similar results have been obtained for all other PDF sets, but the position at which the $b$-quark contribution sets in appears at different $p_{T}$ values.

We conclude that PDF sets with a small value for the $b$-quark threshold should not be used in the GM-VFNS to describe $B$-meson production in the low- $p_{T}$ region. One can try to adjust the definition of the factorization scale $\mu_{i}$ to the threshold $m_{b}^{(\text {thr })}$, but this requires additional fine-tuning, separately for each PDF set, and leads, in some cases, to additional shoulders in the $p_{T}$ distribution which worsens the agreement with the data. This applies to HERAPDF2.0. It would also apply to version 3.0 of the NNPDF PDFs [26]. The NNPDF3.0 set was fitted to data with $m_{b}^{\text {(thr) }}=4.18 \mathrm{GeV}$. The choice of this value was motivated by other determinations of the $b$-quark mass based on QCD sum rules. For NNPDF3.0, $\mu_{i}$ as defined in Eq. (1) is therefore always larger than the PDF threshold $m_{b}^{\text {(thr) }}$, even at $p_{T}=0 \mathrm{GeV}$. We do not show 


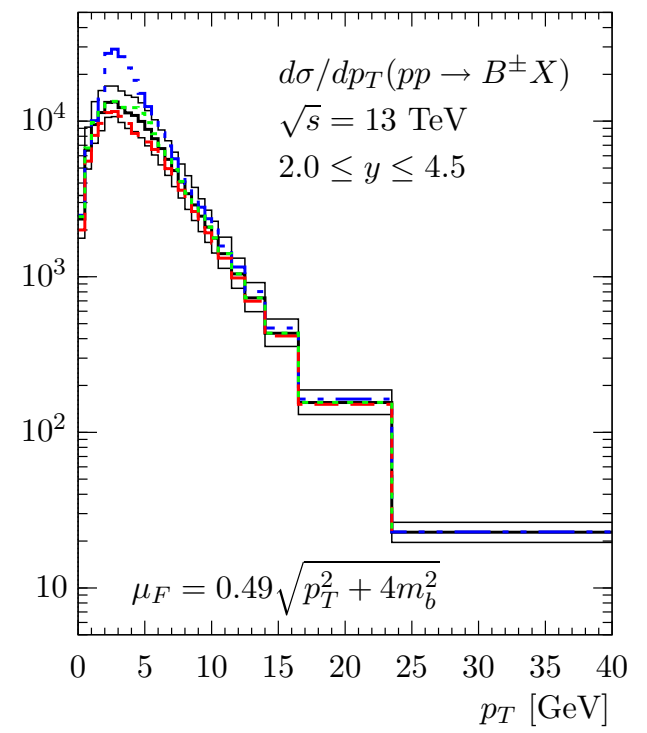

Fig. 1 The $p_{T}$ distribution of $B^{ \pm}$production at $\sqrt{S}=13 \mathrm{TeV}$ integrated over rapidity $2.0 \leq y \leq 4.5$ with $\mu_{F}=0.49 \sqrt{p_{T}^{2}+4 m_{b}^{2}}$, $m_{b}=4.5 \mathrm{GeV}$. Left: Black (full line) histogram: CT14 with $m_{b}^{\text {(thr) }}=$ $4.75 \mathrm{GeV}$; green (short-dashed) histrogram: MMHT with $m_{b}^{\text {(thr) }}=$ $4.75 \mathrm{GeV}$; blue (long-short-dashed) histogram: HERAPDF2.0 with

numerical result for this PDF set because we could not find good agreement with the LHCb data even for very specific choices of the scale parameters.

\section{Cross section results and comparisons with $\mathrm{LHCb}$ data}

The LHCb Collaboration has measured the cross sections for $B^{ \pm}$-meson production at $\sqrt{S}=7$ and $13 \mathrm{TeV}$ in the

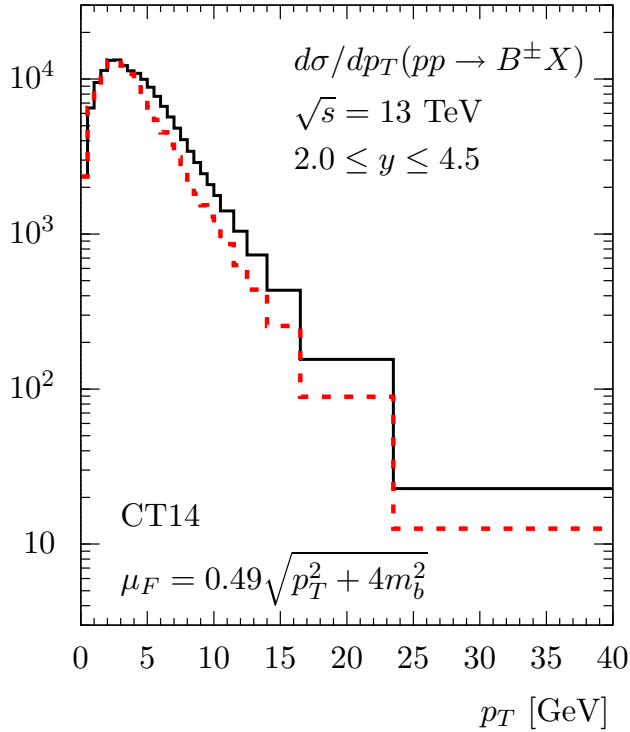

$m_{b}^{\text {(thr) }}=4.5 \mathrm{GeV}$; red (long-dashed) histogram: NNPDF3.1 with $m_{b}^{\text {(thr) }}=4.92 \mathrm{GeV}$. We also show the theory uncertainty band for CT14 (thin black line histogram). Right: The black (full line) histogram shows the complete result for the CT14 PDFs, the red (dashed-line) histogram shows the result without contributions due to $b$ quarks in the initial state

transverse momentum range $0<p_{T}<40 \mathrm{GeV}$ and in five rapidity bins covering $2.0<y<4.5$. The doubledifferential cross section data, $d^{2} \sigma / d y d p_{T}$, as a function of $p_{T}$ and in bins of $y$ are compared with our results for the CT14 PDF set in Fig. 2 (left plot for $\sqrt{S}=7 \mathrm{TeV}$, right plot for $\sqrt{S}=13 \mathrm{TeV}$ ). To improve readability of the plots, both experimental data and theoretical predictions are multiplied by scaling factors $10^{-2}, 10^{-4}, 10^{-6}$ and $10^{-8}$ in the $y$ bins $2.5<y<3.0,3.0<y<3.5,3.5<y<4.0$, and $4.0<y<4.5$, respectively. The full-line histogram is the
Fig. $2 B^{ \pm}$double-differential production cross sections at $\sqrt{S}=7 \mathrm{TeV}$ (left) and $\sqrt{S}=13 \mathrm{TeV}$ (right) as a function of $p_{T}$ and $y$. The black points represent the measured values from the $\mathrm{LHCb}$ Collaboration [20]. The full-line histogram is the prediction with the default choice of scales using the CT14 PDF set. The dashed histograms represent the theoretical uncertainty of the calculated cross sections
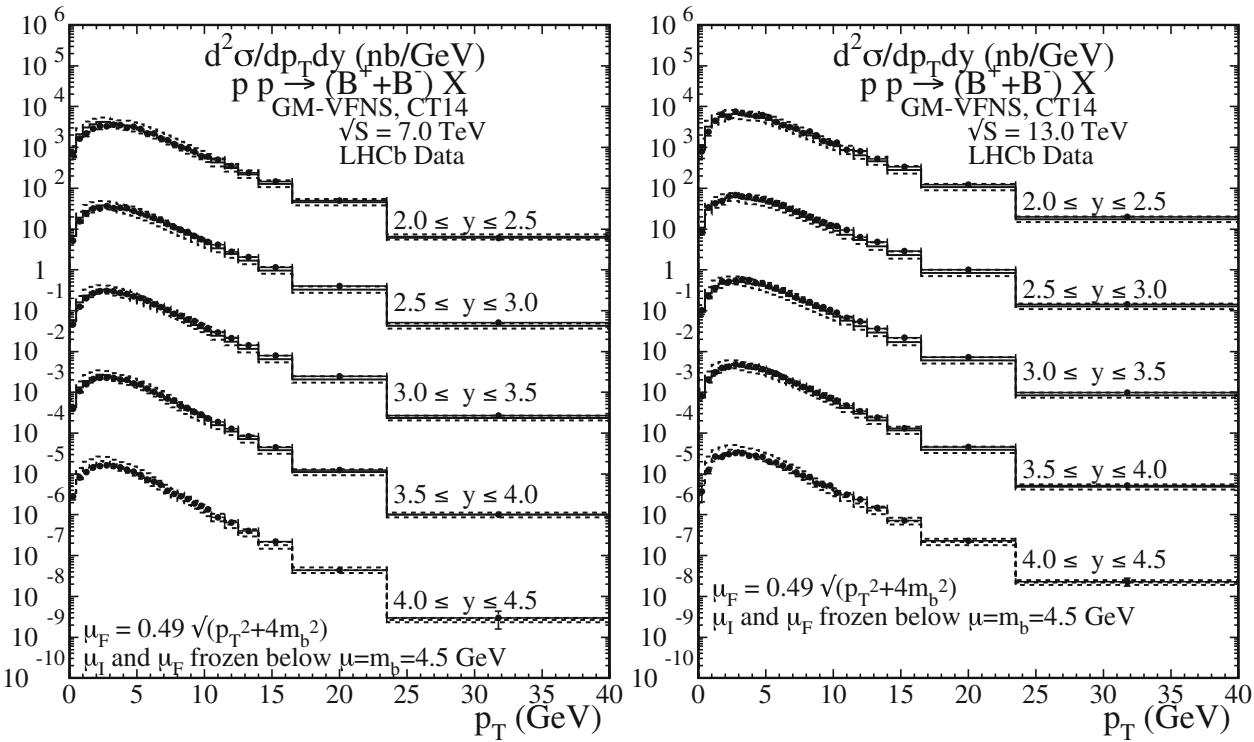
Fig. $3 B^{ \pm}$differential cross sections at $\sqrt{S}=7 \mathrm{TeV}$ (left) and $\sqrt{S}=13 \mathrm{TeV}$ (right) as a function of $p_{T}$, integrated over $y$ in the range $2.0 \leq y \leq 4.5$. The black points represent the measured values from the $\mathrm{LHCb}$ Collaboration. The full-line histogram is the prediction with the default choice of scales using the CT14 PDF set. The dashed histograms represent the theoretical uncertainty of the calculated cross sections
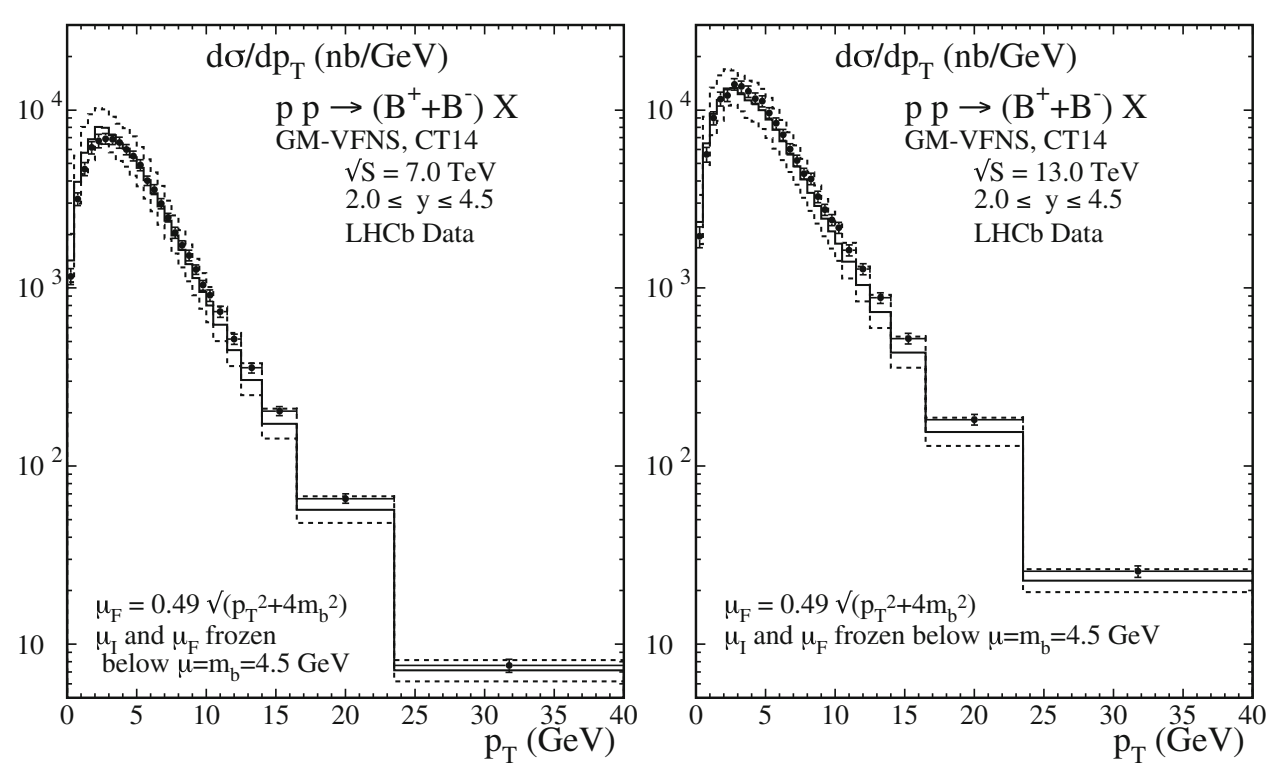

Fig. 4 Ratio of the measured over predicted $B^{ \pm} p_{T}$ distributions at $\sqrt{S}=7 \mathrm{TeV}$ (left) and $13 \mathrm{TeV}$ (right), integrated over $y$ in the range $2.0 \leq y \leq 4.5$. The black points connected by the full-line histogram represent the ratio calculated with the default scales. The error bars represent the experimental uncertainties. The histograms with thin full lines show the theoretical uncertainties obtained by varying the renormalization scale. The dotted histograms show the uncertainty band resulting from a variation of the factorization scale as described in the text. The PDF set CT14 was used

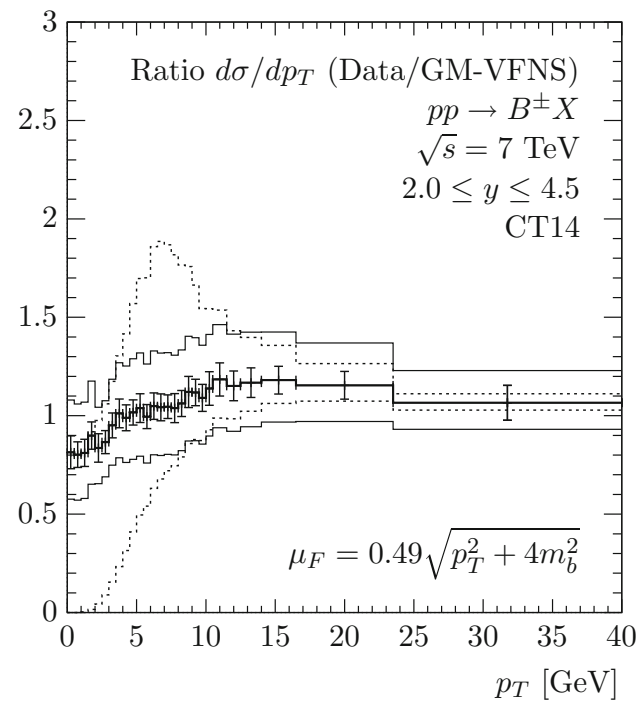

default prediction with the factorization scale $\mu_{F}$ as in Eq. (1) and the renormalization scale $\mu_{R}=\sqrt{p_{T}^{2}+4 m_{b}^{2}}$. The theoretical error is estimated by multiplying $\mu_{R}$ by factors 0.5 and 2.0, but leaving $\mu_{F}$ unchanged. Note that this implies a reduced theoretical error. The results for the maximal and minimal cross sections are given by the dashed histograms. The agreement between data and predictions is excellent, in particular in the small $p_{T}$ range, both for $\sqrt{S}=7$ and $13 \mathrm{TeV}$. As shown in Ref. [20], also the comparison with the FONLL prediction [13] exhibits a similarly good agreement between data and predictions.

In Ref. [20], also the corresponding single differential cross sections $d \sigma / d p_{T}$ obtained from the measured doubledifferential cross sections by summing over the five rapidity bins are given. These data are shown in Fig. 3 and compared with our predictions for $\sqrt{S}=7$ and $13 \mathrm{TeV}$. The agreement between data and predictions is equally good as above for the double-differential cross sections shown in Fig. 2.

The ratios of data over predictions are shown in Fig. 4. From this plot, one can see more clearly the quality of the agreement, which is better than $20 \%$ and well inside the theoretical uncertainties. The majority of the data points agree with the predictions also within the smaller experimental errors; only in the intermediate range $10 \lesssim p_{T} \lesssim 20 \mathrm{GeV}$, the data tend to lie slightly above the predictions. The histograms with thin full lines show the theoretical uncertainties obtained by varying the renormalization scale by a factor 2 up and down. In this figure, we also show the uncertainty band resulting from an additional variation of the factorization scale $\mu_{F}$. Here we follow Ref. [15] and vary $\mu_{F}$ in the 


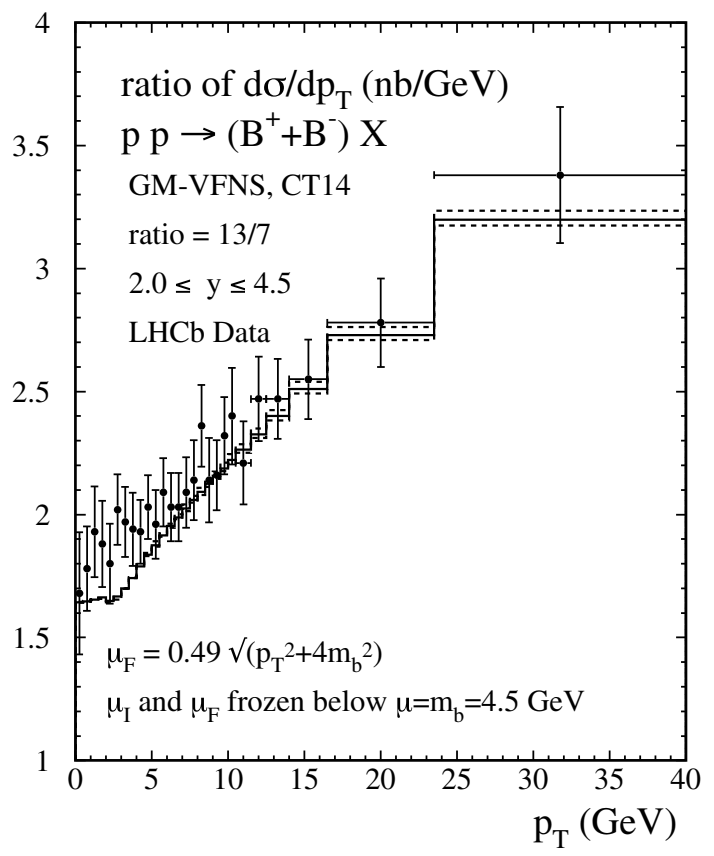

Fig. 5 Ratio of the $B^{ \pm} p_{T}$ distribution at $\sqrt{S}=13 \mathrm{TeV}$ to that at $\sqrt{S}=7 \mathrm{TeV}$, integrated over $y$ in the range $2.0 \leq y \leq 4.5$. The black points represent the measured values from the LHCb Collaboration. The full-line histogram is the prediction with the default choice of scales using the PDF set CT14. The dashed histograms represent the theoretical uncertainties

range between $0.4 \sqrt{p_{T}^{2}+4 m_{b}^{2}}$ (upper dotted histogram) and $0.6 \sqrt{p_{T}^{2}+4 m_{b}^{2}}$ (lower dotted histogram). By increasing the factorization scale, the threshold above which $b$-quark initiated processes contribute is shifted to smaller $p_{T}$ values and the cross section starts to diverge for $p_{T} \rightarrow 0$, i.e. the ratio shown in Fig. 4 becomes small. On the other hand, decreasing the factorization scale moves this threshold to larger $p_{T}$

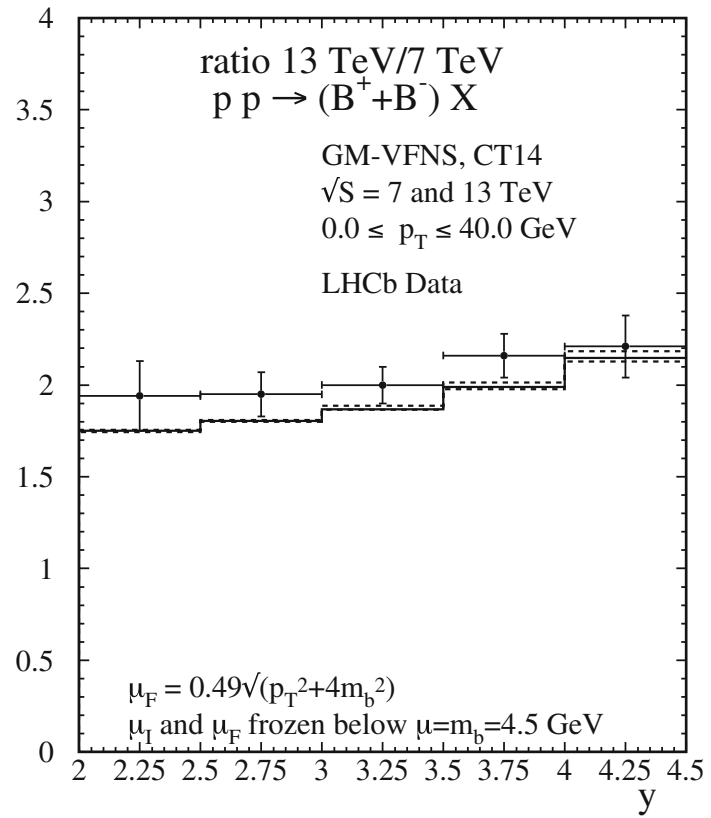

Fig. 7 Ratio $R_{13 / 7}$ of the $B^{ \pm} y$ distributions at $\sqrt{S}=13 \mathrm{TeV}$ and $7 \mathrm{TeV}$, integrated over $p_{T}$ in the range $0 \leq p_{T} \leq 40 \mathrm{GeV}$. The black points represent the measured values from the $\mathrm{LHCb}$ Collaboration. The full-line histogram is the prediction with the default choice of scales using the CT14 PDF set. The dashed-line histograms represent the theoretical uncertainties

and the cross section is suppressed further at intermediate values of $p_{T} \simeq 4-12 \mathrm{GeV}$.

Figure 5 shows the ratio of $d \sigma / d p_{T}$ at the two centerof-mass energies, $R_{13 / 7}=d \sigma(\sqrt{S}=13 \mathrm{TeV}) / d \sigma(\sqrt{S}=$ $7 \mathrm{TeV})$. The data are taken from Ref. [20] and compared with our predictions obtained as the ratios of the cross sections shown in Fig. 3. The comparison shows good agreement. The theoretical uncertainty of the ratio is quite small,
Fig. $6 y$ distribution of $B^{ \pm}$ production at $\sqrt{S}=7 \mathrm{TeV}$ (left) and $13 \mathrm{TeV}$ (right), integrated over $p_{T}$ in the range $0 \leq p_{T} \leq 40 \mathrm{GeV}$. The black points represent the measured values from the $\mathrm{LHCb}$ Collaboration. The full-line histogram is the prediction with the default choice of scales using the CT14 PDF set. The dashed histograms represent the theoretical uncertainties
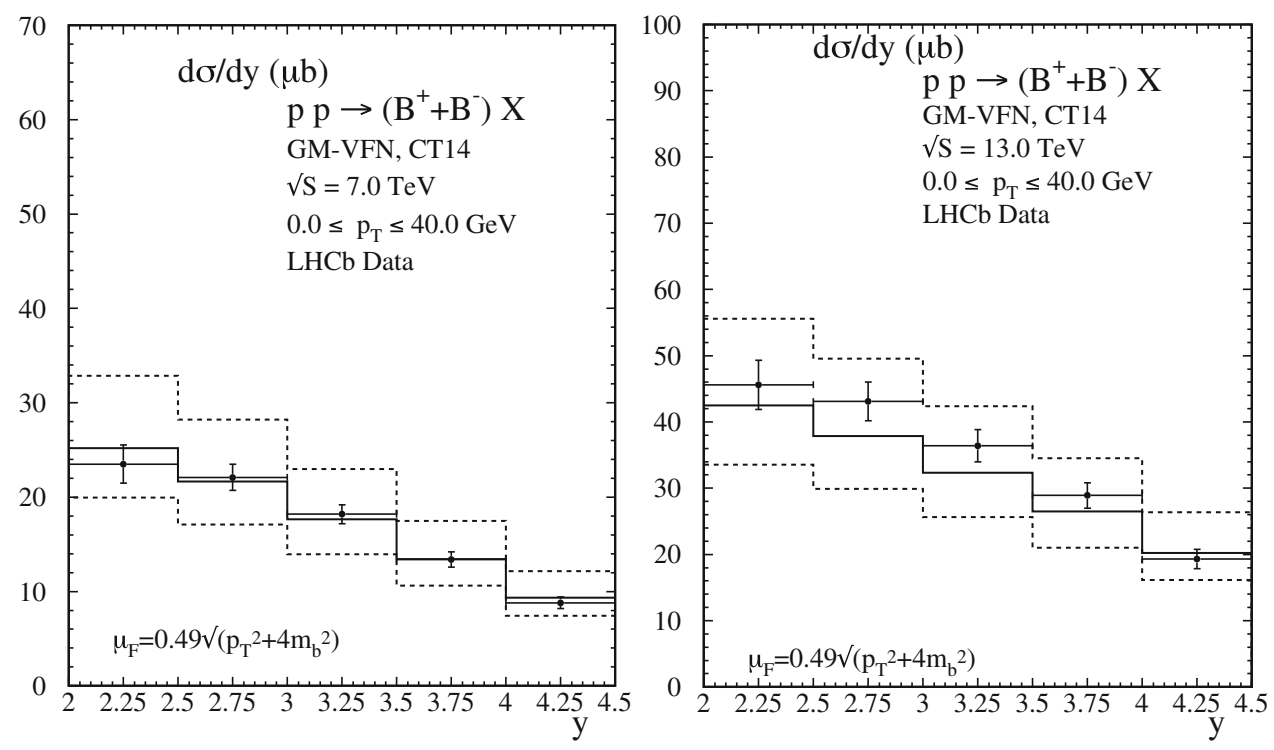
since the scale variation is performed in the numerator and denominator of the ratio in the same way.

Finally we present results for the rapidity distributions. In Fig. 6, we show the cross section $d \sigma / d y$, integrated over the range $0 \leq p_{T} \leq 40 \mathrm{GeV}$, for $\sqrt{S}=7$ and $13 \mathrm{TeV}$ and for the five $y$ bins in the range $2.0<y<4.5$. As expected from the agreement seen for the double-differential cross sections, our predictions of the $y$ dependence also agrees well with the data of the LHCb Collaboration [20]. The ratio $R_{13 / 7}$ as a function of $y$ is shown in Fig. 7. We observe once more good agreement between the data from Ref. [20] and the GM-VFNS predictions.

\section{Conclusions}

We have performed a detailed analysis of $B$-meson production at NLO in the perturbative-QCD framework of the general-mass variable-flavour-number scheme. The recent high-quality data from the LHCb Collaboration can be described in this framework over a large range of transverse momenta, down to $p_{T}=0$. Both the $p_{T}$ and $y$ distributions, as well as the cross section ratios taken at different centerof-mass energies, agree well with data.

We found it to be crucial in this comparison that the transition at small $p_{T}$ values to the fixed-flavour-number scheme is implemented in a proper way. With a judicious choice of the factorization scale it is possible to turn off contributions from initial-state $b$ quarks in the hard-scattering processes. This works, however, only when the heavy-quark threshold in the PDF parametrization matches the corresponding threshold chosen in the FFs. At present, all modern FF sets compatible with the GM-VFNS $[29,34]$ have been obtained with the same fixed value of $m_{b}=4.5 \mathrm{GeV}$. This limits the possible choice of PDFs which lead to a consistent framework. Future improvements of PDF fits will require for more and more precise data to be included, and it is expected that inclusive heavy-quark production will play an important role for that. It may become important in this challenge to consider both PDFs and FFs on the same footing within a common fit to data.

Acknowledgements B. A. K. was supported in part by the German Federal Ministry for Education and Research (BMBF) through Grant no. 05H18GUCC1. We thank M. V. Garzelli for useful discussions.

Data Availability Statement This manuscript has no associated data or the data will not be deposited. [Authors' comment: Numerical results of theoretical predictions can be obtained upon request. Experimental data shown in the figures for comparison can be found in the given references.]

Open Access This article is distributed under the terms of the Creative Commons Attribution 4.0 International License (http://creativecomm ons.org/licenses/by/4.0/), which permits unrestricted use, distribution, and reproduction in any medium, provided you give appropriate credit to the original author(s) and the source, provide a link to the Creative Commons license, and indicate if changes were made. Funded by SCOAP ${ }^{3}$.

\section{References}

1. D. Acosta et al. [CDF Collaboration], Phys. Rev. D 71, 032001 (2005). arXiv:hep-ex/0412071

2. A. Abulencia et al. [CDF Collaboration], Phys. Rev. D 75, 012010 (2007). arXiv:hep-ex/0612015

3. A.M. Sirunyan et al. [CMS Collaboration], Phys. Rev. Lett. 119, 152301 (2017). arXiv:1705.04727 [hep-ex]

4. G. Aad et al. [ATLAS Collaboration], JHEP 1310, 042 (2013). arXiv:1307.0126 [hep-ex]

5. V. Khachatryan et al. [CMS Collaboration], Phys. Rev. Lett. 106, 112001 (2011). arXiv:1101.0131 [hep-ex]

6. S. Chatrchyan et al. [CMS Collaboration], Phys. Rev. Lett. 106, 252001 (2011). arXiv:1104.2892 [hep-ex]

7. S. Chatrchyan et al. [CMS Collaboration], Phys. Rev. D 84, 052008 (2011). arXiv:1106.4048 [hep-ex]

8. R. Aaij et al. [LHCb Collaboration], JHEP 1204, 093 (2012). arXiv: 1202.4812 [hep-ex]

9. R. Aaij et al. [LHCb Collaboration], JHEP 1510, 172 (2015). Erratum: [JHEP 1705 (2017) 063] arXiv:1509.00771 [hep-ex]

10. V. Khachatryan et al. [CMS Collaboration], Phys. Lett. B 771, 435 (2017). arXiv:1609.00873 [hep-ex]

11. S. Chatrchyan et al. [CMS Collaboration], Phys. Lett. B 714, 136 (2012). arXiv:1205.0594 [hep-ex]

12. R. Aaij et al. [LHCb Collaboration], Chin. Phys. C 40, 011001 (2016). arXiv:1509.00292 [hep-ex]

13. M. Cacciari, S. Frixione, N. Houdeau, M.L. Mangano, P. Nason, G. Ridolfi, JHEP 1210, 137 (2012). arXiv:1205.6344 [hep-ph]

14. B.A. Kniehl, G. Kramer, I. Schienbein, H. Spiesberger, Phys. Rev. D 84, 094026 (2011). arXiv:1109.2472 [hep-ph]

15. B.A. Kniehl, G. Kramer, I. Schienbein, H. Spiesberger, Eur. Phys. J. C 75, 140 (2015). arXiv:1502.01001 [hep-ph]

16. B.A. Kniehl, G. Kramer, I. Schienbein, H. Spiesberger, Phys. Rev. D 71, 014018 (2005). arXiv:hep-ph/0410289

17. B.A. Kniehl, G. Kramer, I. Schienbein, H. Spiesberger, Eur. Phys. J. C 41, 199 (2005). arXiv:hep-ph/0502194

18. I. Helenius, H. Paukkunen, JHEP 1805, 196 (2018). arXiv: 1804.03557 [hep-ph]

19. R. Aaij et al. [LHCb Collaboration], JHEP 1308, 117 (2013). arXiv:1306.3663 [hep-ex]

20. R. Aaij et al. [LHCb Collaboration], JHEP 1712, 026 (2017). arXiv:1710.04921 [hep-ex]

21. M. Benzke, M.V. Garzelli, B. Kniehl, G. Kramer, S. Moch, G. Sigl, JHEP 1712, 021 (2017). arXiv:1705.10386 [hep-ph]

22. M. Benzke, M.V. Garzelli, B.A. Kniehl. arXiv:1812.02248 [hep$\mathrm{ph}]$

23. S. Dulat et al., Phys. Rev. D 93, 033006 (2016). arXiv:1506.07443 [hep-ph]

24. H. Abramowicz et al. [H1 and ZEUS Collaborations], Eur. Phys. J. C 75, 580 (2015). arXiv:1506.06042 [hep-ex]

25. L.A. Harland-Lang, A.D. Martin, P. Motylinski, R.S. Thorne, Eur. Phys. J. C 75, 204 (2015). arXiv:1412.3989 [hep-ph]

26. R.D. Ball et al. [NNPDF Collaboration], JHEP 1504, 040 (2015). arXiv: 1410.8849 [hep-ph]

27. R.D. Ball et al. [NNPDF Collaboration], Eur. Phys. J. C 77, 663 (2017). arXiv:1706.00428 [hep-ph]

28. A. Buckley, J. Ferrando, S. Lloyd, K. Nordstöm, B. Page, M. Rüfenacht, M. Schönherr, G. Watt, Eur. 
Phys. J. C 75, 132 (2015). arXiv:1412.7420 [hep-ph]. http://projects:hepforge.org/lhapdf/pdfsets

29. B.A. Kniehl, G. Kramer, I. Schienbein, H. Spiesberger, Phys. Rev. D 77, 014011 (2008). arXiv:0705.4392 [hep-ph]

30. A. Heister et al. [ALEPH Collaboration], Phys. Lett. B 512, 30 (2001). arXiv:hep-ex/0106051

31. G. Abbiendi et al. [OPAL Collaboration], Eur. Phys. J. C 29, 463 (2003). arXiv:hep-ex/0210031

32. K. Abe et al. [SLD Collaboration], Phys. Rev. Lett. 84, 4300 (2000). arXiv:hep-ex/9912058
33. K. Abe et al. [SLD Collaboration], Phys. Rev. D 65, 092006 (2002). Erratum: [Phys. Rev. D 66 (2002) 079905] arXiv:hep-ex/0202031

34. M. Salajegheh, S.M. Moosavi Nejad, H. Khanpour, B.A. Kniehl, M. Soleymaninia, Phys. Rev. D 99, 114001 (2019). arXiv:1904.08718 [hep-ph]

35. J. Abdallah et al. [DELPHI Collaboration], Eur. Phys. J. C 71, 1557 (2011). arXiv:1102.4748 [hep-ex] 